\title{
Introduction for the special issue in honor of Chris Godsil
}

\author{
Aleksandar Jurišićc ${ }^{1}$ - Akihiro Munemasa ${ }^{2}$. \\ Simone Severini ${ }^{3,4}$
}

Received: 30 March 2016 / Accepted: 30 March 2016 / Published online: 11 April 2016

(C) Springer Science+Business Media New York 2016

Chris Godsil was born in Bendigo, Australia, in 1949 and grew up in the small town of Warragul near Melbourne. He finished undergraduate studies in biochemistry at the University of Melbourne in 1969 and then worked for four years as a biochemist before continuing with his graduate studies, which were also at the University of Melbourne. His interests were now in mathematics, and he worked under the supervision of Derek Holton, a combinatorialist. He finished his $\mathrm{PhD}$ in 1979 with the dissertation Graphs with Regular Groups. Chris then spent almost two years in Austria at the Montanuniversität Leoben, where he worked with Wilfried Imrich. This was followed by a move to Canada, where he joined the Mathematics Department at Simon Fraser University in Burnaby, British Columbia. In 1987, Chris moved to Waterloo, Ontario, to join the Department of Combinatorics and Optimization $(\mathrm{C} \& \mathrm{O})$, at the University of Waterloo.

Chris has served at $\mathrm{C} \& \mathrm{O}$ very well over the past 29 years, as an instructor of a wide variety of undergraduate and graduate courses, as the department's most active graduate student supervisor (14 MMath and $14 \mathrm{PhD}$ ), as Associate Chair for Graduate

\footnotetext{
$\bowtie \quad$ Akihiro Munemasa

munemasa@math.is.tohoku.ac.jp

Aleksandar Jurišić

aj@fri.uni-lj.si

Simone Severini

simoseve@gmail.com

1 Faculty of Computer and Information Science, Večna pot 113, Ljubljana 1000, Slovenia

2 Graduate School of Information Sciences, Tohoku University, Aoba-ku, Sendai 980-8579, Japan

3 Department of Computer Science, University College London, Gower Street, London WC1E 6BT, UK

4 United Kingdom and Institute of Natural Sciences, Shanghai Jiao Tong University, 5F, Pao Yue-Kong Library, No. 800 Dongchuan Road, Minhang District, Shanghai 200240, China
} 
Studies, as Department Chair, and has influenced multiple generations of mathematicians. He has extremely high standards and demands the best from his students. At the same time, he is encouraging, supportive, and very conscientious about developing his students into capable and independent research mathematicians. In 2014, he received the University of Waterloo Award of Excellence in Graduate Supervision.

Chris is a research leader in discrete mathematics and has been (and continues to be) hugely influential in many different fields of mathematics around the world. His extensive contributions to mathematical research include three very successful books, Algebraic Combinatorics, Algebraic Graph Theory (co-authored with Gordon Royle), The Erdôs-Ko-Rado Theorem: Algebraic Approaches (co-authored with Karen Meagher), and over 100 papers in research journals (which have a total of over one thousand citations). He is a cofounder of the Journal of Algebraic Combinatorics and serves on the editorial board of several other journals, including Australasian Journal of Combinatorics, Journal of Combinatorial Theory Series B, and Combinatorica.

One central theme in Chris's research is to determine useful relations between the properties of a graph and algebraic properties of the various adjacency matrices. This branch of graph theory, known as algebraic graph theory, has been tremendously successful and is very active. For example, the founders of Google used eigenvectors of the webgraph to create their profoundly successful search engine. Eigenvalues of graphs are also used in testing graph isomorphism and for graph drawing. The smallest eigenvalue of a graph gives information about the size of the independent sets in the graph, and recent work by Chris has also shown that the structural information about these independent sets can be found in the eigenspaces. In 2012, Brouwer and Haemers published a book in this area called Spectra of Graphs.

Chris has also worked extensively on the famous Erdős-Ko-Rado (EKR) theorem. This theorem is a result in extremal set theory that was published in 1961, and in the fifty years since then, this result has become a cornerstone of extremal set theory. Many researchers are developing extensions, generalizations, and applications of this result; this is a very active area of research that is related to several different areas of mathematics. One of Chris's major contributions is a new algebraic method to prove these types of theorems; not only does this once again prove the power of algebraic tools, but it gives a way to connect the different versions of the EKR theorem.

More recently, Chris has been a pioneer in using techniques from algebraic combinatorics to tackle some fundamental questions in quantum information theory. There have been a surprising number of problems related to quantum computing and quantum information theory that are naturally looked at from the viewpoint of algebraic graph theory. In relation to this context and the broader framework of quantum information and communication, Chris laid the mathematical foundations of information transfer on spin systems and their control theory. He contributed to the problem of quantum state determination and proposed new directions in the study of non-local games.

These topics: algebraic graph theory, Erdős-Ko-Rado theorems and quantum information theory, served as the central themes of the conference "Algebraic Combinatorics: Spectral Graph Theory, Erdős-Ko-Rado Theorems and Quantum Information Theory," held at the University of Waterloo from June 23-27, 2014. One hundred twenty-seven mathematicians from around the world gathered to participate in the conference to celebrate Chris Godsil's work (and also his 65th birthday), which was 
co-organized by his ex-graduate students and postdocs: Ada Chan (York University), Aleksandar Jurišić (University of Ljubljana), Bill Martin (Worcester Polytechnic Institute), Karen Meagher (University of Regina), Mike Newman (University of Ottawa), and the $\mathrm{C} \& \mathrm{O}$ Department Chair Alfred Menezes. The present issue contains selected papers written by invited speakers of this conference.

The conference program, in its balance of combinatorial and algebraic themes with such a variety of applications, served not only as a conduit for collaboration among established researchers, but also as a concise survey of a broad array of research challenges for students and new PhDs. 